\title{
Testing the hypothesis of tolerance strategies in Hiatella arctica L. (Mollusca: Bivalvia)
}

Received: 15 April 2004/ Revised: 14 February 2005/ Accepted: 14 February 2005/Published online: 6 April 2005

(C) Springer-Verlag and AWI 2005

\begin{abstract}
The physiological and biocenotic optima of Hiatella arctica L. inhabiting shallow water fouling communities of the White Sea were compared. The biomass and proportion of $H$. arctica in communities were used for the estimation of biocenotic optima or community success. The physiological state of populations was assessed by means of the fluctuating asymmetry. The fluctuating asymmetry of $H$. arctica was calculated using the valve weights. It was determined that the shell of $H$. arctica possesses a slight directional asymmetry, the right valve usually being larger (and heavier) than the left one. The relationship between fluctuating and directional asymmetries is discussed. High biomass and proportion of $H$. arctica in the community generally correspond with high levels of fluctuating asymmetry. Thus, a discrepancy between physiological and ecological optima is observed, which is recognised as being characteristic of a tolerance strategy. However, in the absence of pressure from major filter-feeding competitors such as the mussel Mytilus edulis L. and the solitary ascidian Styela rustica L., populations of $H$. arctica possess a high biomass and low levels of fluctuating asymmetry. It appears that $H$. arctica prefers to inhabit shelters or depressions, or to be covered by other organisms. Populations of $H$. arctica existing in the absence of shelter had extremely high levels of fluctuating asymmetry despite the absence of filter-feeding competitors. Thus, the strategy adopted by $H$ arctica $\mathrm{L}$. in the investigated upper $5 \mathrm{~m}$ layer of water in the White Sea can be described as a stress- and competitor-tolerant strategy.
\end{abstract}

Communicated by E. Rachor

V. V. Khalaman

The White Sea Biological Station, Zoological Institute of the Russian Academy of Sciences, Universitetskaya nab., 1, 199034, St. Petersburg, Russia

E-mail: kha@onego.ru

Tel.: + 7-812-1140097

Fax: + 7-812-1140444
Keywords Fluctuating asymmetry $\cdot$ Directional asymmetry $\cdot$ Hiatella arctica $\cdot$ Biofouling .

Tolerance strategy $\cdot$ The White Sea

\section{Introduction}

Ramenskii (1935) described three main survival strategies in terrestrial plants: tolerance $(\mathrm{S})$, ruderal $(\mathrm{R})$ and competitive $(\mathrm{K})$ strategies. This classification was adopted in the Russian literature, but received little attention elsewhere. Almost 40 years later the classification of survival strategies was "rediscovered" by Grime (1974), became widely adopted by plant ecologists, and was then developed further. Tolerance strategies were divided into two categories, referring to plants that are tolerant to unfavourable abiotic environmental conditions and to a high degree of competitive pressure from other species, respectively (Mirkin and Rosenberg 1983). Another classification scheme of tolerance strategies was suggested by Campbell and Grime (1992) who distinguished species following a disturbance (D) - and a stress-tolerance (S) strategy, respectively.

It appears that the classification of plant survival strategies also may be applied to sedentary marine animals. Knowledge of the distribution of these strategies among populations may provide a useful explanation of succession patterns in benthic and fouling communities. Studies on the succession of fouling communities in the White Sea indicate that the survival strategies of fouling organisms indeed are similar to those of plants (Sirenko et al. 1978; Oshurkov 1985, 1992; Khalaman 1989, 2001a,b).

Hiatella arctica, a widespread bivalve, is one of the main fouling organisms in the White Sea, and research into its competitive strategy is required in order to understand its population dynamics and role in fouling communities. In contrast to mussels, $H$. arctica does not form a uniform fouling community: The 
settlements of $H$. arctica are usually interspersed by those of other species. At least within the upper 3-5 m water layer, $H$. arctica, although numerous, is not the sole dominant of most fouling communities (Oshurkov 1985; Kulakovskii et al. 1993; Khalaman 1998, 2001a).

The distribution patterns of $H$. arctica in the upper water layer in the White Sea, and the proportion of this molluse in fouling communities, appear to be the result of a tolerance strategy. The presence of large filter-feeding competitors such as mussels (Mytilus edulis L.) and solitary ascidians (Styela rustica L.) in shallow-water fouling communities in the White Sea is widely documented. Thus, the strategy of $H$. arctica might be expected to be both stress- and competitortolerant.

It has been suggested that the physiological and biocenotic (community success) optima in species with tolerance strategies do not coincide (Lopatin 1963; Vasilevich 1987). Thus, tolerant species might be expected to attain a high biomass and abundance rank within a community where the environmental factors are suboptimal for this species and vice versa. Therefore, there is a strong need to compare the biocenotic success and the physiological state of $H$. arctica in different communities.

The biomass and abundance rank of $H$. arctica within communities reflect the biocenotic or community success of the population. The physiological state of the population can be estimated using the fluctuating asymmetry (FA) of the shells, i.e., random deviations from perfect bilateral symmetry. FA is the most widely used measure of developmental stability, which in turn is a sensitive indicator of the physiological state of individuals within a population. In other words, developmental stability refers to the ability of a developing organism to produce a consistent phenotype in a given environment (Leary and Allendorf 1989; Palmer and Strobeck 1992; Freeman et al. 1993; Graham et al. 1993a; Palmer 1996; Møller 1997). In recent years, FA has been used extensively as an indicator of genetic and environmental stress at the population level and to estimate the prevailing environmental conditions (Alados et al. 1993; Graham et al. 1993a; Møller et al. 1995; Bonn et al. 1996; Vollestad and Hindar 1997; Carrascal et al. 1998; Lajus and Sukhotin 1998; Smith 1998; Lajus 2001; and many others). Thus, high levels of FA in a population of $H$. arctica would indicate sub-optimal environmental conditions and vice versa.

The main goal of the present study was to test the hypothesis that $H$. arctica $\mathrm{L}$. adopts a tolerance strategy within shallow-water fouling communities in the White Sea. It was decided to carry out statistical analyses of bilateral valve asymmetry FA as an indicator of the environmental conditions under which the population exists. Based on information and on data of biomass and abundance rank, the tolerance strategy of $H$. arctica was assessed.

\section{Methods}

Sampling and laboratory procedures

$H$. arctica and fouling communities containing this species were sampled from artificial substrata in mussel culture farms situated in Kandalaksha Bay in the White Sea in July-August 1997 and August-September 1998 (Fig. 1). Vertically suspended substrata (nylon rope or fish net ribbon) in the upper water layer are used for cultivation of blue-mussels (M. edulis L.) in the White Sea. The length of the artificial substrata is $3 \mathrm{~m}$ (Kulakowskii and Kunin 1983). Besides the mussel community, some other fouling organisms such as ascidians ( $S$. rustica), sponges (Halichondria panicea) and $H$. arctica can develop on the artificial substrata of culture farms (Oshurkov 1992; Khalaman 2001a). The location of the
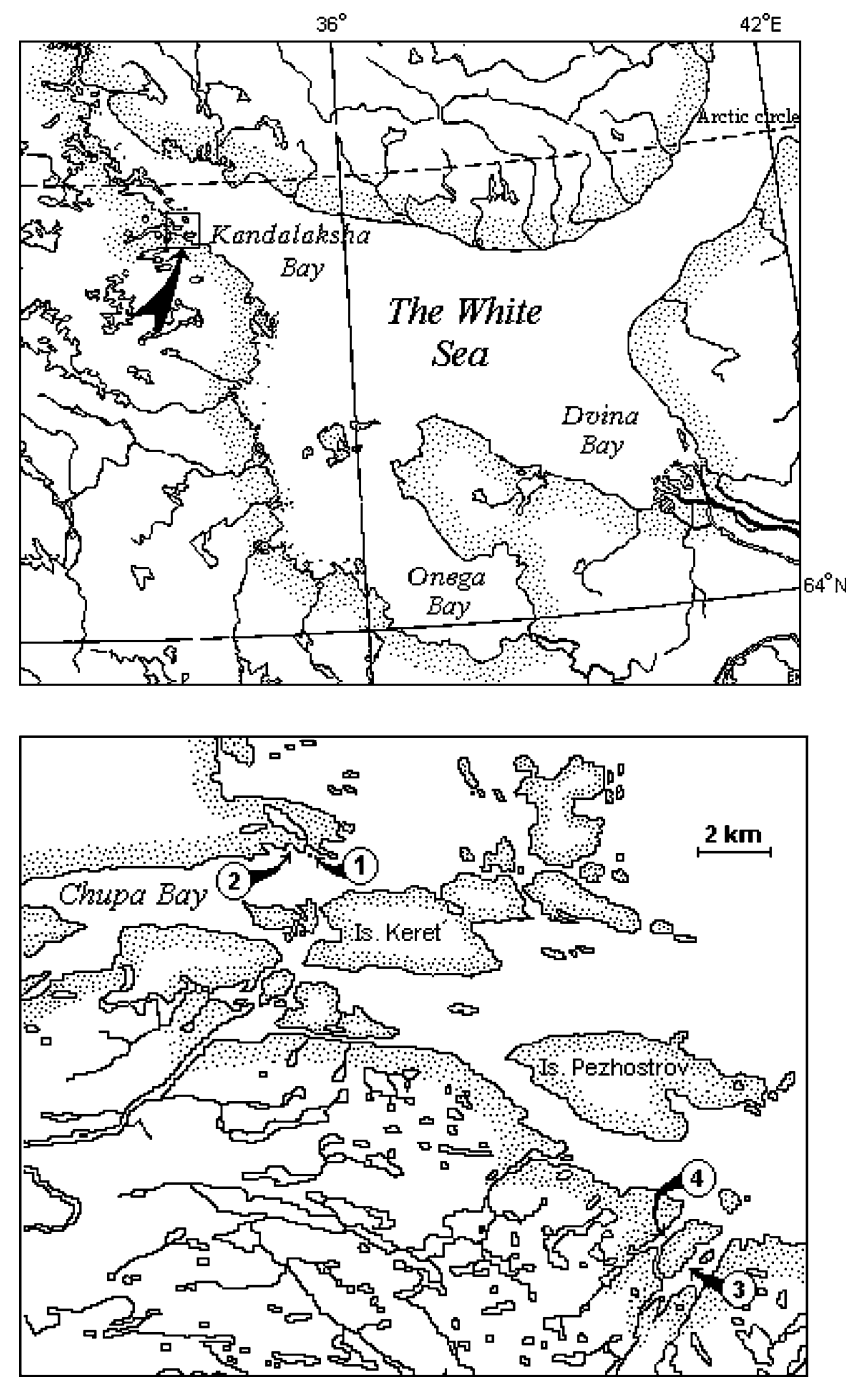

Fig. 1 Study area and location of mussel culture farms where sampling was carried out; 1 Krivozjorskaya Bay, 2 Kruglaya Inlet, 3 Nikolskaya Inlet, 4 Osechkova Inlet 
mussel culture farm, volume of sampled material and types of fouling community are given in Table 1 .

Sampling of all species present in the fouling communities was carried out at nine stations. For each sample, artificial substrate (ribbon of fish net) of $0.1 \mathrm{~m}$ length was taken, with all associated organisms. At four stations, only specimens of $H$. arctica were picked from the fouling communities. In these cases, the type of fouling community was determined visually. At four additional stations, specimens of $H$. arctica were picked from vertically suspended experimental plastic plates measuring $0.5 \times 0.7 \mathrm{~m}$. Sampling of the entire fouling community was not carried out from these plates. Where the entire community was sampled, the numbers of $H$. arctica necessary for statistical analysis of FA were taken from these samples. However, at two stations (nos. 4 and 5; see Table 1) with extremely low densities of $H$. arctica, it was necessary to pick additional specimens from neighbouring areas of the same fouling community. The number of replicate samples per station ranged between two and five, dependent on the length of the substrate occupied by a homogenous fouling community. Sorting, species identification, counting of specimens and biomass determinations were carried out in the laboratory. Wet weight biomass of the communities sampled and of $H$. arctica populations were converted to units per $1 \mathrm{~m}$ length of substrate. After weighing, the FA of $H$. arctica was analysed for each sample.

The relative weight of the two valves of $H$. arctica, subsequently referred to as the "trait", was used to estimate FA. Approximately 50 individuals of $H$. arctica were taken from each population (see Table 1). The shells of $H$. arctica are fragile, and the extremely thin outer part of the valves is often broken during handling. However, such slight damage does not significantly influence the weight, while length measurements are greatly affected by shell injury. Nonetheless, badly damaged specimens were excluded from the analyses. To dissolve soft tissue, molluscs were immersed in a $5 \%$ solution of $\mathrm{NaOH}$ for $24 \mathrm{~h}$ at $40^{\circ} \mathrm{C}$. The shells were then rinsed in fresh water and the remaining soft tissue was removed. All valves were numbered, air-dried until a constant weight was attained and weighed to the nearest $0.5 \mathrm{mg}$.

\section{Numerical analyses}

Data were subjected to linear regression and correlation analyses. Parameters for linear regressions were calculated out by both least squares and maximum likelihood methods (Casella and Berger 1990). Where both variables show random variation, the second method is more appropriate for regression analysis. There is solid criticism against using the least squares regression technique for asymmetry analysis (Graham et al. 1998). For this reason, the subsequent data analysis was based on the maximum likelihood technique.

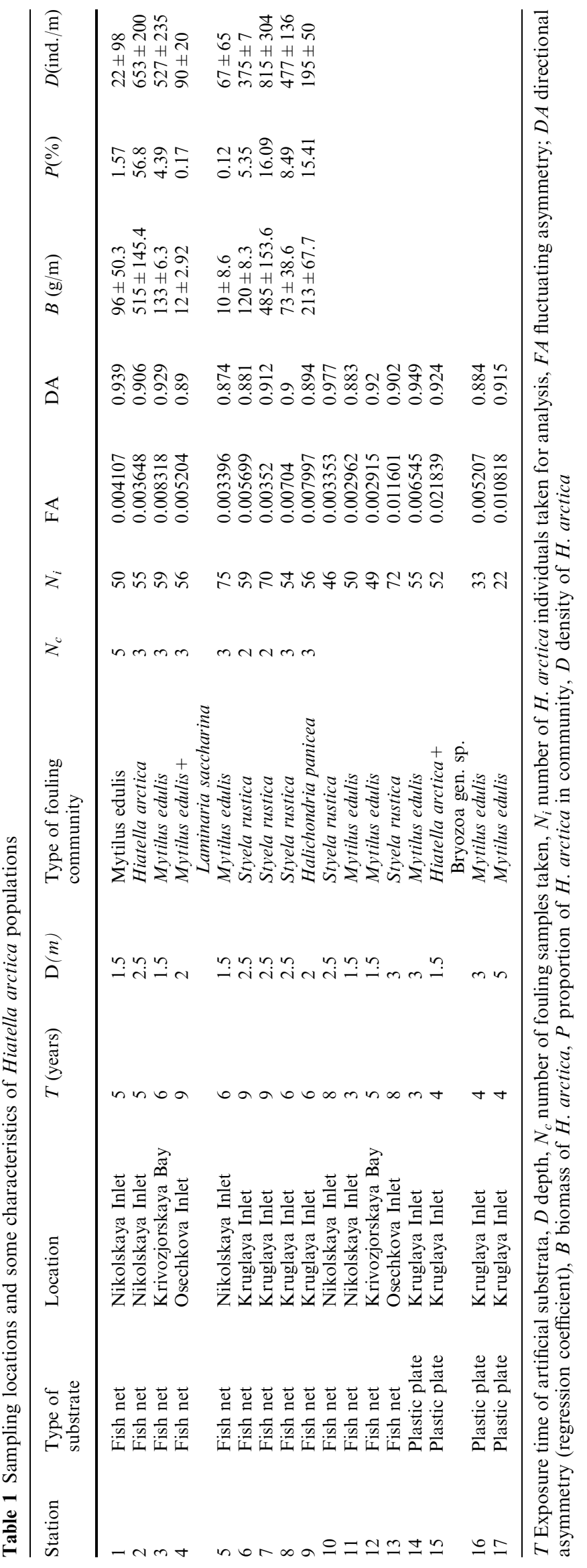


The analysis of FA was based on the following reasoning: The FA of bilaterally symmetrical animals can be expressed as the variance of individual asymmetries $\left(d_{i}\right)$ : FA $=\operatorname{Var}\left(d_{i}\right)$ with $d_{i}=r_{i}-l_{i}$, where $r_{i}$ is the dimension of the trait on the right side of individual $i$, and $l_{i}$ is the dimension of the same trait on the left side of individual $i$. The average value of $d_{i}$ should be zero, if the right and left sides are symmetrical.

Directional asymmetry (DA) appears if the average of $d_{i}$ is not equal to zero (Graham et al. 1993b, 1998; Palmer 1996). This means that the dimension of the trait on one side is, on average, larger than on the other side. In this case, individual asymmetries are the result of both FA $\left(d_{i \mathrm{FA}}\right)$ and DA $\left(d_{i \mathrm{DA}}\right)$ :

$d_{i}=d_{i \mathrm{FA}}+d_{i \mathrm{DA}}$

Let us assume that the weight of the right valve $(\mathrm{Wr})$ is an independent variable and that the relationship between the left and right valves is expressed by a linear equation. Then,

$W l_{\exp }=a+b W r$,

where $W l_{\text {exp }}$ is the theoretical expected weight of the left valve at a given weight of the right part of the shell $(\mathrm{Wr})$.

If the intercept $(a)$ does not differ from zero and the regression coefficient $(b)$ is equal to $1, \mathrm{DA}$ is absent. When the right valve is heavier than the left valve, the regression coefficient is less than 1 . In this case, the absolute difference between the opposite valves is dependent on the size of the mollusc. If $b=1$, but $a$ does not equal zero, the difference between the valves is constant.

From $d_{i}=W r-W l_{i}$, and $d_{i}=d_{i \mathrm{FA}}+d_{i \mathrm{DA}}$ follows

$d_{i \mathrm{FA}}+d_{i \mathrm{DA}}=W r_{i}-W l_{i}$

It is essential to note that $d_{i \mathrm{DA}}$ in fact denotes the difference between the observed weight of the right valve $\left(W r_{i}\right)$ and the theoretically predicted weight of the left valve $\left(W l_{\text {iexp }}\right)$ :

$d_{i \mathrm{DA}}=W r_{i}-W l_{i \exp }$.

Hence, Eq. 1 may be expressed as:

$d_{i \mathrm{FA}}+\left(W r_{i}-W l_{i \exp }\right)=W r_{i}-W l_{i}$,

which is equivalent to

$d_{i \mathrm{FA}}=W l_{i \exp }-W l_{i}$

Equation 2 allows a simple calculation of individual FA. In the present study, the FA was estimated as the normalised to the expected weight of left valve $W l_{\text {exp. }}$. variance of $d_{i \mathrm{FA}}$ :

$\mathrm{FA}=\operatorname{Var}\left(d_{i \mathrm{FA}} / W l_{\mathrm{exp}}\right)$

In the literature, the use of total residual variance as an estimation of FA is recommended (Graham et al. 1998). However, since total residual variance computed by the maximum likelihood method is closely associated with the average size of the tested object (weight of shells in this case), the method is here considered inappropriate for this purpose. There was a high correlation $(r=0.83)$ between total residual variance and the weight of the shell in $H$. arctica. For correct assessment of FA and the relationship between FA and the ecological success of $H$. arctica, it is essential to avoid these autocorrelation effects. Thus, the present study uses a new method which allows an assessment of FA independent of shell weight.

\section{Results and discussion}

Differences between right and left valves

In the literature, it is often stated that shells of $H$. arctica L. have equally sized valves (Skarlato 1987). In reality, the right valve of this mollusc commonly is somewhat larger than the left one and weighs more. However, specimens of $H$. arctica with a larger left valve can also be found, although in the present study, the percentage of these individuals per sample did not exceed $24 \%$. In the populations studied, differences between the relative weight of the valves were maximally $40 \%$, with an average of $10 \%$. In some individuals, there were no demonstrable differences in valve weights. However, the number of such individuals was comparatively low $(<10 \%)$.

The distribution of the differences in weight between right and left valve normalised to the average weight of a valve $\left(D_{i}\right)$ is shown in Fig. 2.

$D_{i}=(W r-W l) /[(W r-W l) / 2]$.

The distribution differs from normal only in having a significant positive kurtosis $(k=2.5)$. The average of $D_{i}$ does not equal zero but is positive, with a value of 0.095 and $\mathrm{SD}=0.076(n=913)$. Thus, the specimens of $H$. arctica studied possess a slight DA, with the right part of the shell being normally larger than the left one.

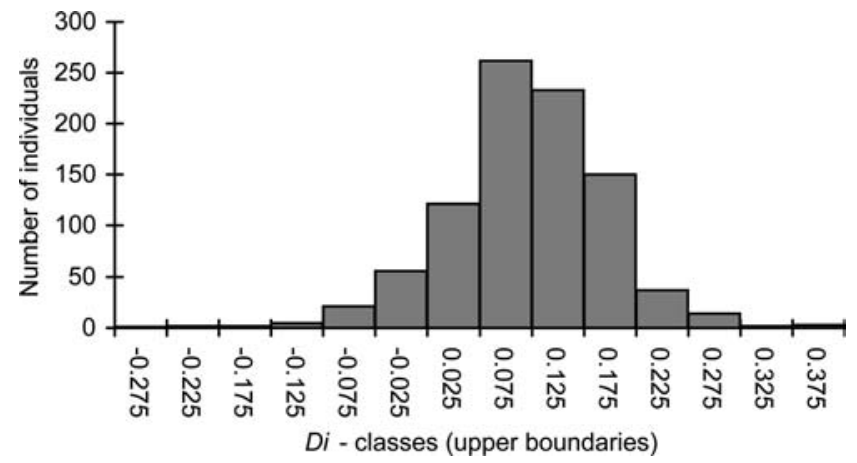

Fig. 2 Frequency distribution of the differences in weight between right and left valves normalised to the average weight of a valve: $D_{i}=(W r-W l) /[(W r+W l) / 2]$ 
Analysis of directional asymmetry

The relationship between the weights of the left and right valves is well described by a linear regression. All samples show a correlation coefficient $(r)$ of not less than 0.99 . No significant differences were observed between results obtained by the least squares technique and the maximum likelihood technique. This is illustrated by the high correlation $(r=0.96)$ between regression coefficients obtained by the different techniques. Moreover, the results of the investigation as a whole do not depend on the chosen method of calculation. This is likely to reflect the very close relationship between right and left valves.

The regression coefficient $(b)$ varies from 0.87 to 0.98 for different settlements of $H$. arctica. Calculated for pooled data, the value is 0.906 . Intercept $(a)$ fluctuates slightly around zero from -0.0044 to +0.0036 for different samples, and is almost equal to zero (0.0002) for pooled data. Thus, in the present study, the level of DA is assessed in terms of the regression coefficient which was calculated for each population of $H$. arctica separately. It must be borne in mind at this point that the regression coefficient is inversely related to DA.

\section{Analysis of fluctuation asymmetry}

FA varies from 0.0029 to 0.0218 for different settlements of $H$. arctica. The highest value was detected for populations of $H$. arctica inhabiting one of the four investigated plastic plates (station 15, see Table 1). This plate was submerged at $1.5 \mathrm{~m}$ depth and was covered by a poor settlement of different bryozoans and a welldeveloped population of $H$. arctica. At this station, there was no organic or inorganic shelter available to the molluscs. It must be noted that the preference of $H$. arctica for shelters or depressions or for being covered by other organisms has prompted some authors to consider this species as a drilling mollusc (Zevina 1972), although this is unlikely due to the fragile nature of the shell. The high value of FA in the Hiatella population at station 15 probably was caused by the absence of suitable shelters. As is evident from Table 1, settlements of $H$. arctica located on other plates which were covered by a well-developed mussel fouling population (stations 14, 16 and 17) show a moderate level of FA.

Relationship between the proportion of "left-valve" individuals and directional and fluctuating asymmetries

As stated above, the proportion of individuals where the left valve is larger than the right one (left-valve individuals) varied from 0 to $24 \%$ among the sampling stations. It is clear that an increase in the proportion of left-valve individuals can result from either an increase in FA, or a decrease of DA, or a combination of both.

The relationship between the proportion of left-valve specimens and the regression coefficient is shown in

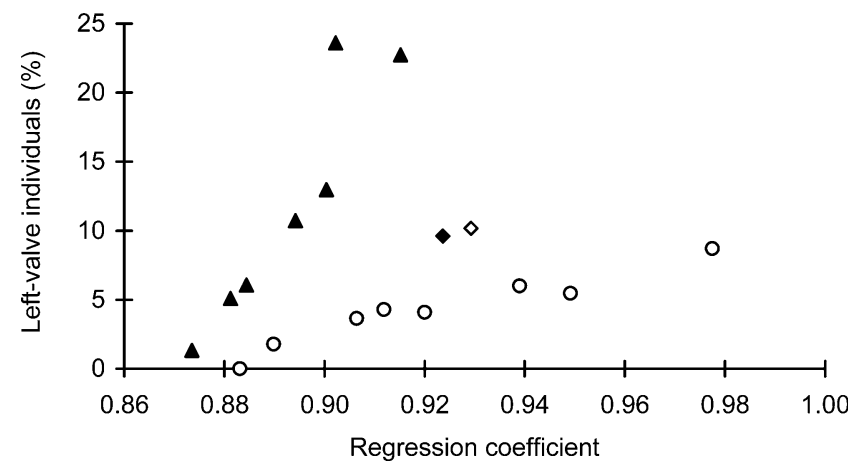

Fig. 3 Proportion of left-valve individuals vs regression coefficient of the relationship between left and right valves in populations of $H$. arctica. Symbols as follows: empty circles and solid triangles-first and second groups of $H$. arctica populations, respectively; empty and solid rhombus - intermediate populations

Fig. 3. On average, with an increased proportion of leftvalve specimens, the regression coefficient also increases and, therefore, the DA declines. This result is clear and as might have been expected. However, the dispersion of the data points is extremely high. Moreover, there seem to be two different groups of $H$. arctica settlements: one of them, marked on Fig. 3 and subsequent figures by empty circles, shows a comparatively low proportion of left-valve molluscs. Even slight increases in the proportion of left-valve individuals corresponds to considerable decreases in DA, with the regression coefficient tending to 1 . In the other group of settlements, marked by solid triangles, sharp increases in the proportion of left-valve individuals correspond with only slight increases in the regression coefficient, i.e., with slight decreases in DA. The regression coefficient does not reach such high values as in the other group. As can be seen from Fig. 4, the first group of settlements does not show a close relationship between FA and the proportion of left-valve individuals, whereas there is a high positive correlation between these parameters in the second group. Thus, both processes which can result in an increase in the proportion of left-valve individuals (i.e., increase in FA and decrease in DA, respectively) is demonstrated in the

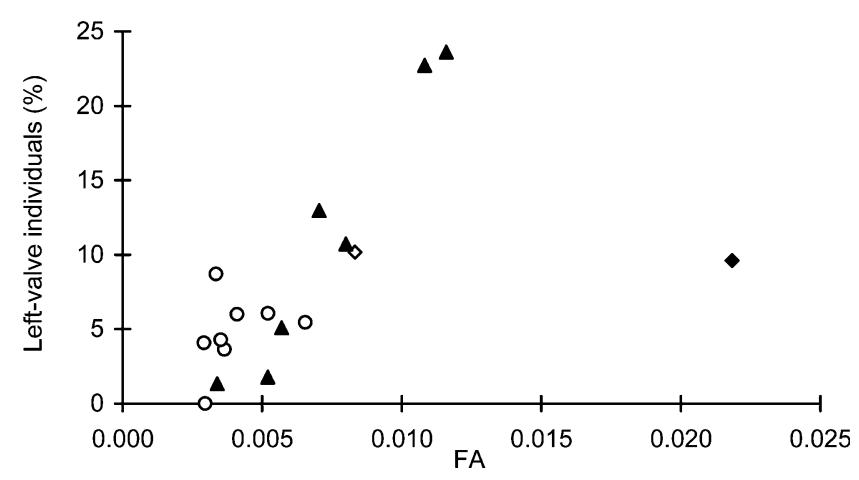

Fig. 4 Relationship between the proportion of left-valve individuals and the fluctuating asymmetry of shells in populations of H. arctica (symbols as in Fig. 3) 
present study, although a certain degree of overlap is evident.

The clear separation of the two groups of Hiatella settlements is interesting (see Fig. 3). Only two intermediate points are evident. The first one (solid rhombus) represents a $H$. arctica settlement with an extremely high FA value, located on a plastic plate (station 15). The second one (empty rhombus) refers to a settlement with an unusual history of development (station 3). In this area, there was a low initial colonisation and growth rate of mussels ( $M$. edulis), and thus s low competition pressure for settled $H$. arctica. However, during the last 2 years prior to sampling, secondary fouling of juvenile mussels had taken place on this substrate and extensively covered the settlement of $H$. arctica. It is highly probable that the comparatively high FA observed for this population of $H$. arctica is due to these events. The level of DA and the proportion of left-valve individuals in both settlements appear to be closely linked.

The relationship between the regression coefficient (inverse estimation of DA) and FA is shown in Fig. 5. Two discrete types of H.arctica populations can be distinguished: Within the first group (empty circles), there is a high variation of the regression coefficient, but the variance in FA was low. FA remained comparatively low and independent of the regression coefficient. The second group (solid triangles) shows a positive linear relationship between the regression coefficient and FA. The general patterns is that FA increases (and thus DA declines) with increasing regression coefficients. However above regression coefficients of 0.9 , FA shows a tendency to remain constant or even to decrease. It should be noted that the maximum of FA (except for one outlier) approximately corresponds to the regression coefficient calculated for pooled data (0.906).

The observed relationship between fluctuating and directional asymmetries suggests that the deviation from a hypothetical normal level of DA is a response to a decrease in FA. If this is the case, it is proposed that the estimation of developmental instability should involve both FA and the deviation of DA from its hypothetical normal level. However, this proposal is in conflict with the widely accepted opinion that DA is developmentally

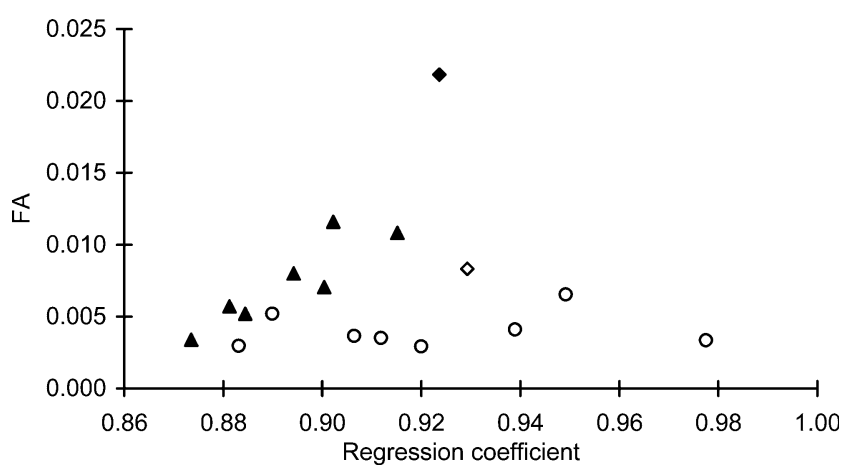

Fig. 5 FA of shells versus the coefficient of regression between left and right valves (symbols as in Fig. 3) controlled and cannot be used to evaluate developmental instability (Van Valen 1962; Palmer and Strobek 1992, 1997; Palmer 1996). The data of the present study do not allow an unequivocal rejection of the above hypothesis, because DA is not constant among populations. In addition, populations of $H$. arctica with extreme values of DA show low levels of FA. Thus, the present results support the view that DA may in some cases reflect developmental instability (Leary and Allendorf 1989; Graham et al. 1993b; Møller 1994).

Some additional questions arise from the proposed interaction between fluctuating and directional asymmetries: e.g., how can the "normal" level of DA be determined and how can a combination of FA and DA be used to assess development instability? However, addressing these issues is beyond the scope of the present work and remains for future studies. Therefore, for the time being, only FA is used as an indicator of development instability.

Relationships between ecological characteristics of $H$. arctica settlements and directional and fluctuating asymmetries of populations

A clear relationship between the type of fouling community and FA or DA of $H$. arctica was not observed. The relationships between the biomass of $H$. arctica and its proportion in fouling communities, respectively, and
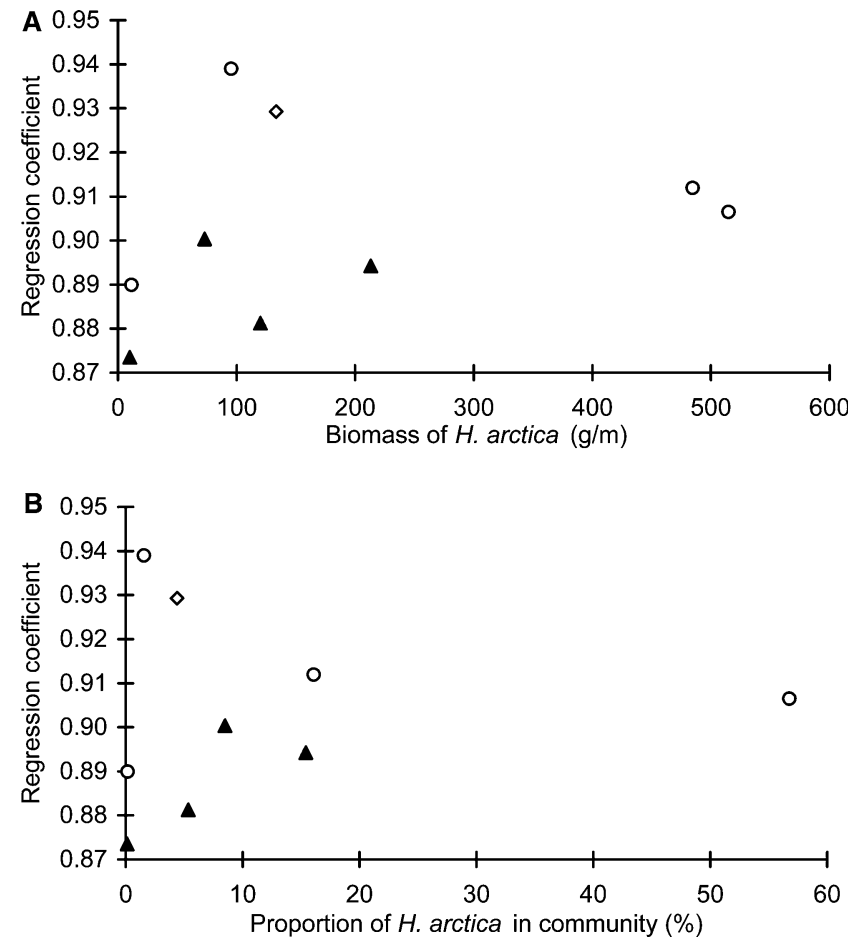

Fig. 6 Coefficient of regression between left and right valves versus some characteristics of $H$. arctica populations: A biomass of $H$. arctica, B proportion of $H$. arctica in community (symbols as in Fig. 3) 
the regression coefficient are shown in Fig. 6. The regression coefficient for each community approximates the value calculated for pooled data (0.906) as biomass (or the proportion of $H$. arctica within the community) increases.

FA generally increases with increasing biomass of $H$. arctica, but two populations with an extremely high biomass show low values of FA (Fig. 7a). A similar relationship exists between the proportion of $H$. arctica in the community and FA (Fig. 7b). Populations of $H$. arctica show a low values of FA both when the biomass of $H$. arctica is extremely high and low (Fig. 7a).

How do the data obtained correspond to the predicted stress tolerance strategy in $H$. arctica? Species which are tolerant to extreme abiotic factors, will develop high biomasses in such extreme environments, due to the absence of less tolerant competitors. In spite of high community success, the physiological state of the tolerant species may be low due to the fact that the environmental regime is sub-optimal. Apparently, the tolerant species is expelled by competitors from optimal to sub-optimal environments. Species which are tolerant to the impact of competitors demonstrate an analogous relationship between community success and the physiological state of the population. However, the explanation for this is different from that given for species tolerant to extreme abiotic factors.

$H$. arctica settles among numerous and often large filter-feeding competitors such as the blue mussel
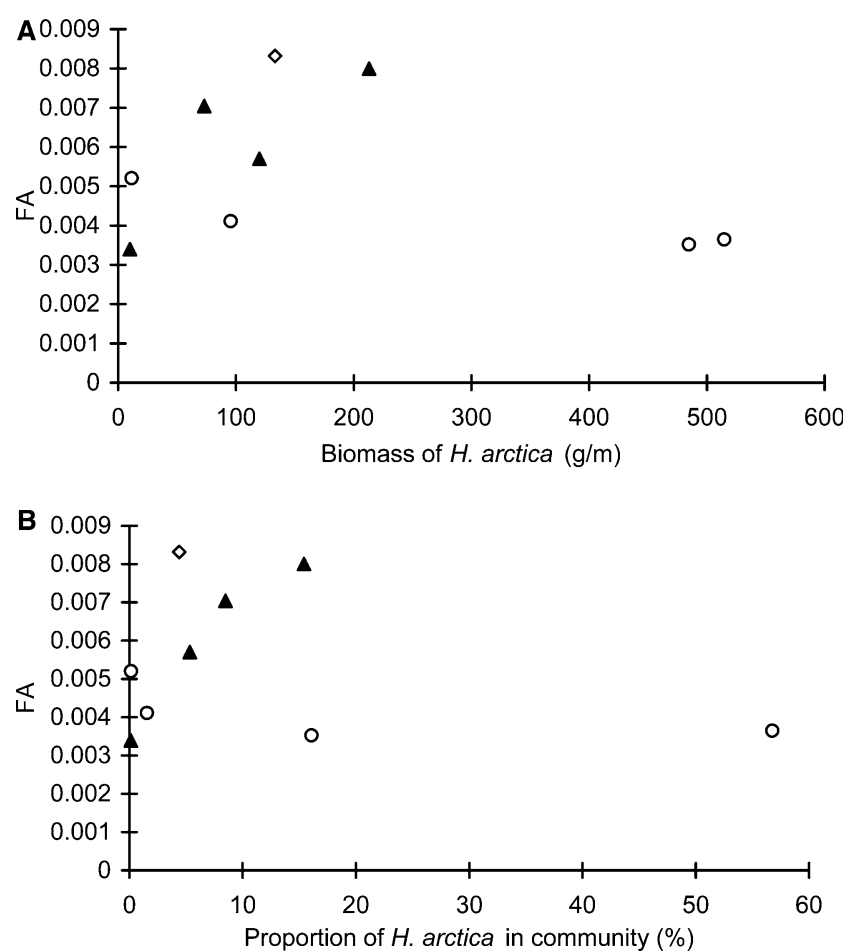

Fig. 7 Relationships between the fluctuating asymmetry of shells and some characteristics of $H$. arctica populations: A biomass of $H$. arctica, B proportion of $H$. arctica in community (symbols as in Fig. 3)
$M$. edulis or the solitary ascidian $S$. rustica. Under competitive stress, the resources (food and space) can support only very restricted abundances of tolerant species (low biomass but high physiological state of population). If the abundance of a population increases, the intraspecific competition for limiting resources also increases, resulting in a decline of the physiological state of organisms (high biomass but low physiological state of population). Thus, a low physiological state and, consequently, a high level of FA are the cost for ecological success. This phenomenon is illustrated by the left parts of the graphs in Fig. 7.

However, the settlements of $H$. arctica with the highest proportion of the community and an extremely high biomass showed low levels of FA (Fig. 7). It should be noted that these high values may not reflect the species' competitive ability, but rather the absence of competitors. For example, as a result of wave action, patches of mussels drop down from artificial substrata to the bottom, while $H$. arctica remains on the substratum, released from the high competitive pressure by $M$. edulis. This can be seen in Fig. 8, where the relationships between community biomass and biomass of $H$. arctica are shown. Even after removal of mussels, $H$. arctica is not deprived of shelter. It is unlikely that all sedentary organisms disappear from the substrata simultaneously, and in addition, the artificial substrate (fish net) itself may serve as shelter. In the absence of competitors, populations of any species develop as rapidly as resources allow. In the absence of large filterfeeding competitors, food access is not a limiting factor for $H$. arctica. This might explain why the biomass of this mollusc attained so high a value and why this process was not accompanied by a corresponding increase in FA. Such a situation can be observed when the proportion of $H$ arctica within the community exceeded approximately $15 \%$ (Fig. 7).

The extremely high level of FA that was measured for one of the $H$. arctica populations inhabiting plastic plates (station 15) where mussels were missing, additionally supports the suggestion that this species requires some form of shelter (organic or inorganic). In spite of absence of the competitors, the environmental

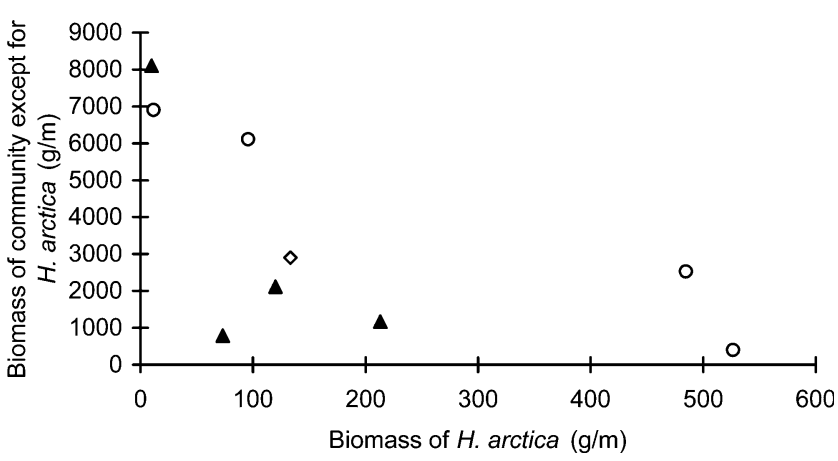

Fig. 8 Relationship between the biomass of fouling communities (except for $H$. arctica) and the biomass of $H$. arctica (symbols as in Fig. 3) 
conditions at this site were apparently unfavourable for $H$. arctica. Populations of $H$. arctica inhabiting plates that were covered by mussels (stations 14, 16 and 17) had only moderate FA values.

At to the "normal" levels of DA, there are three main reasons to suggest 0.906 as an estimation of the normal DA value of $H$. arctica in the White Sea. First, the regression coefficient of the relationship between left and right valves calculated for pooled data is equal to 0.906 . Second, $H$. arctica inhabiting most optimal environmental conditions (without major competitors) have a similar regression coefficient. Finally, the turning point of the relationship between directional and fluctuating asymmetries is close to this DA value. If 0.906 is a correct estimation of the normal DA value, methods for the use of joint FA and DA parameters can be developed. For this purpose, the regression parameters obtained for pooled data were also applied to calculate FA for each population of $H$. arctica, but it is not yet known whether this approach is the most appropriate. The relationship between this joint parameter and the biomass of $H$. arctica is shown in Fig. 9. The relationship was not markedly different from that resulting from separate parameters (see Fig. 7a), but was less pronounced.

The same species can exhibit different strategies depending on the environmental biotic conditions (Grime 1979; Mirkin and Rosenberg 1983; Vasilevich 1987). The present study shows $H$. arctica to display a tolerance strategy in shallow waters in the White Sea, where the species is not dominant within the community. In deeper waters, the species may adopt a different survival strategy. This is indicated by the observation that $H$. arctica can dominate in fouling communities at $10 \mathrm{~m}$ and deeper in the White Sea (Oshurkov 1985). In addition, it is interesting to note the dominance of $H$. arctica within certain benthic communities in Arctic seas (Filatova 1957; Gulliksen et al. 1980).

A summer thermocline is known to develop in the inlets of the White Sea, which commonly lies at depths between 5 and $20 \mathrm{~m}$, depending on the individual hydrological characteristics of the bays (Naumov et al. 1986; Babkov 1995). In deeper and cooler water, there is

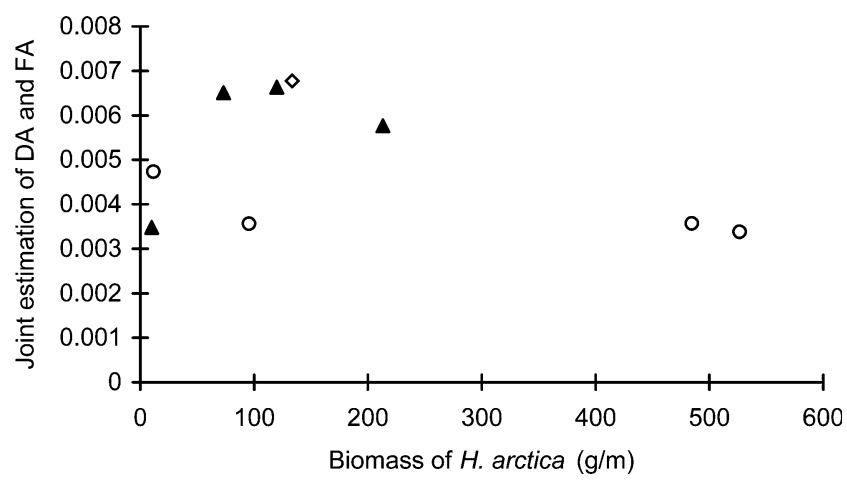

Fig. 9 Relationship between the suggested joint parameter of directional and fluctuating asymmetries and the biomass of $H$. arctica (symbols as in Fig. 3) a marked decline in the abundance of H.arctica's main competitor, M. edulis, and the role of $H$. arctica in such communities differs from that in shallower waters.

Measurement of FA appears to be a comparatively easy, and thus attractive, method for estimating the physiological state of populations, particularly in ecological studies. However, as the present study indicates, this approach is not yet sufficiently developed theoretically as well as methodically. The heated discussions, which still exist in the literature, is clear evidence of this point (Palmer 1996; Møller 1997; Palmer and Strobeck 1997; Clarke 1998; Lajus 2001). Nevertheless, as with all scientific disciplines, such controversy is a normal and necessary step in the development of the technique.

The present study cannot be considered as definite evidence that $H$. arctica L. follows a stress- and competitor-tolerant strategy in shallow waters in the White Sea. The conclusion is based on some more or less proven assumptions: (1) the triangular model of plant survival strategies can be applied to sedentary marine animals, (2) the level of FA reflects the physiological state of population and (3) the physiological and biocenotic optima in species that adopt tolerance strategies do not coincide.

The study was an attempt to test whether these assumptions applied to a concrete species. The results indicate that this may be case in the studied populations of $H$. arctica, and that these really adopt tolerance strategies. However, the additional studies on $\mathrm{H}$. arctica and other species are needed.

Acknowledgements The author is very grateful to Sabine Cochrane for her assistance in preparing this paper and for her kind help with the English. I acknowledge the advice and critical comments on the manuscript by Dmitry Lajus. I also thank Ann Merete Hjelset for her continuous help during the work on the article. This study was supported by the Norwegian Research Council and complies with the current laws of Russia and Norway.

\section{References}

Alados CL, Escos J, Emlen JM (1993) Developmental instability as an indicator of environmental-stress in the Pacific hake (Merluccius productus). Fish Bull 91:587-593

Babkov AI (1995) Peculiarities of the hydrological regime of small inlets in Kandalaksha and Onega Bays. In: Scarlato OA (ed) White Sea. Biological resources and problems of their rational exploitations. Part 1 (in Russian). Zoological Institute Russian Academy of Sciences, St. Petersburg, pp 51-61

Bonn A, Gasse M, Rolff J, Martens A (1996) Increased fluctuating asymmetry in the damselfly Coenagrion puella is correlated with ectoparasitic water mites: implications for fluctuating asymmetry theory. Oecologia 108:596-598

Campbell BD, Grime JP (1992) An experimental test of plant strategy theory. Ecology 73:15-29

Carrascal LM, Senar JC, Mozetich I, Uribe F, Domenech J (1998) Interactions among environmental stress, body condition, nutritional status, and dominance in great tits. Auk 115:757738

Casella G, Berger RL (1990) Statistical inference. Brooks/Cole, Pacific Grove, CA

Clarke M (1998) Developmental stability and fitness: the evidence is not quite so clear. Am Nat 152:762-766 
Filatova ZA (1957) Review of Bivalvia fauna for North Seas of USSR (in Russian). Proc Oceanol Inst USSR Acad Sci 20:3-59

Freeman DC, Graham JH, Emlen JM (1993) Developmental stability in plants - symmetries, stress and epigenesis. Genetica 89:97-119

Graham JH, Freeman DC, Emlen JM (1993a) Developmental stability: a sensitive indicator of populations under stress. In: Landis WG, Hughes JS, Lewis MA (eds) Environmental toxicology and risk assessment, ASTM STP 1179. American Society for Testing and Materials, Philadelphia, pp 136-158

Graham JH, Freeman DC, Emlen JM (1993b) Antisymmetry, directional asymmetry, and dynamic morphogenesis. Genetica 89:121-137

Graham JH, Emlen JM, Freeman DC, Leamy LJ, Kieser JA (1998) Directional asymmetry and the measurement of developmental instability. Biol J Linn Soc 64:1-16

Grime JP (1974) Evidence for the existence of three primary strategies in plants and its relevance to ecological and evolutionary theory. Am Nat 111:1169-1194

Grime JP (1979) Plant strategies and vegetation processes. Wiley, New York

Gulliksen B, Haug T, Sandnes OK (1980) Benthic macrofauna on new and old lava grounds at Jan Mayen. Sarsia 65:137-148

Khalaman VV (1989) Investigation of succession of fouling communities using the information index of species diversity (in Russian). Proc Zool Inst USSR Acad Sci 203:34-45

Khalaman VV (1998) Correlations of the spatial distribution of organisms in fouling communities of the White Sea (in Russian with English summary). J Gen Biol (Zhurnal Obschey Biologii) 59:58-73

Khalaman VV (2001a) Fouling communities developing at the mussel culture farms in the White Sea (in Russian with English summary). Biol Morya 27:268-278

Khalaman VV (2001b) Succession of fouling communities developing at the mussel culture farms in the White Sea. Biol Morya 27:399-406 (in Russian with English summary)

Kulakowskii EE, Kunin BL (1983) The theoretical basis of mussel cultivation in the White Sea (in Russian). Nauka, Leningrad

Kulakowskii EE, Sukhotin AA, Khalaman VV (1993) The formation of cultured mussel populations in different points of Chupa inlet (Kandalaksha bay) (in Russian with English summary). Proc Zool Inst USSR Acad Sci 253:24-41

Lajus DL (2001) Variation patterns of bilateral characters: variation among characters and among populations in the White Sea herring, Clupea pallasi marisalbi (Berg) (Clupeidae, Teleosti). Biol J Linn Soc 74:237-253

Lajus DL, Sukhotin AA (1998) A new approach for the assessment of stochastic variation: analysis of behavioural response in blue mussel (Mytilus edulis L.). Helgol Wiss Meeresunters 52:141145

Leary RF, Allendorf FW (1989) Fluctuating asymmetry as an indicator of stress: implication for conservation biology. Trends Ecol Evol 4:214-217

Lopatin VD (1963) On questions about the interactions between cenotypes of edificator plants and their areas (in Russian with English summary). Rep Acad Sci USSR 148:956-957
Møller AP (1994) Directional selection on directional asymmetry-testes size and secondary sexual characters in birds. Proc R Soc Lond Ser B Biol Sci 258:147-151

Møller AP (1997) Developmental stability and fitness: a review. Am Nat 149:916-932

Møller AP, Sanotra GS, Vestergaard KS (1995) Developmental stability in relation to population-density and breed of chickens Gallus gallus. Poult Sci 74:1761-1771

Mirkin BM, Rosenberg GS (1983) Dictionary of modern phytocenology with definitions (in Russian). Nauka, Moscow

Naumov AD, Babkov AI, Fedyakov VV (1986) Bottom communities of the Kolvitsa Inlet of the Kandalaksha Bay of the White Sea. In: Ecological investigations of bottom organisms of the White Sea (in Russian). Zool Inst USSR Acad Sci, Leningrad, pp 91-122

Oshurkov VV (1985) Dynamics and structure of some fouling and benthos communities in the White Sea. In: Biofouling ecology in the White Sea (in Russian). Zool Inst USSR Acad Sci, Leningrad, pp 44-59

Oshurkov VV (1992) Succession and climax in some fouling communities. Biofouling 6:1-12

Palmer AR (1996) Waltzing with asymmetry. Is fluctuating asymmetry a powerful new tool for biologists or just an alluring new dance step? Bioscience 46:518-531

Palmer AR, Strobeck C (1992) Fluctuating asymmetry as a measure of development stability: implications of non-normal distributions and power of statistical tests. Acta Zool Fenn 191:137-144

Palmer AR, Strobeck C (1997) Fluctuating asymmetry and developmental stability: heritability of observable variation vs. heritability of inferred cause. J Evol Biol 10:39-49

Ramenskii LG (1935) On the principal points, main concepts and terms using in plant ecology (in Russian). Sov Bot 4:25-42

Sirenko BI, Kunin BL, Oshurkov VV, Kataeva TK, Babkov AI, Golikov AN, Khlebovich VV, Kulakowskii EE (1978) Succession of fouling communities on artificial substrata in the White Sea. In: Spatial distribution and ecology of coastal byocenoses (in Russian). Nauka, Leningrad, pp 10-13

Skarlato OA (ed) (1987) Molluscs of the White Sea. Keybooks of USSR fauna published by the Zoological Institute of USSR Acad Sci. Nauka, Leningrad

Smith LH (1998) Asymmetry of early paleozoic trilobites. Lethaia 31:99-112

Van Valen L (1962) A study of fluctuating asymmetry. Evolution $16: 125-142$

Vasilevich VI (1987) Types of plant strategies and phytocoenotypes (in Russian with English summary). J Gen Biol (Zhurnal Obschey Biologii) 48:368-375

Vollestad LA, Hindar K (1997) Developmental stability and environmental stress in Salmo salar (Atlantic salmon). Heredity $78: 215-222$

Zevina GB (1972) Biofouling in the seas of USSR. Moscow State University, Moscow 